\title{
Oral versus intravenous iron therapy in patients with inflammatory bowel disease and iron deficiency with and without anemia in Germany - a real-world evidence analysis
}

This article was published in the following Dove Press journal: ClinicoEconomics and Outcomes Research

\author{
Jürgen Stein ${ }^{1,2}$ \\ Jennifer Scarlet $\mathrm{Haas}^{3}$ \\ Siew Hwa Ong ${ }^{4}$ \\ Kathrin Borchert ${ }^{3}$ \\ Thomas Hardt ${ }^{5}$ \\ Elmira Lechat ${ }^{4}$ \\ Kerry Nip ${ }^{5}$ \\ Douglas Foerster ${ }^{4}$ \\ Sebastian Braun ${ }^{3}$ \\ Daniel C Baumgart ${ }^{6}$ \\ 'Interdisciplinary Crohn Colitis \\ Center Rhein-Main, Frankfurt/ \\ Main, Germany; ${ }^{2}$ Department \\ of Gastroenterology and \\ Clinical Nutrition, DGD Clinics \\ Sachsenhausen, Teaching Hospital \\ of the J.W. Goethe University, \\ Frankfurt/Main, Germany; ${ }^{3}$ Xcenda \\ GmbH, Hannover, Germany; ${ }^{4}$ Vifor \\ Pharma Ltd., Glattbrugg, Switzerland; \\ ${ }^{5}$ Vifor Pharma Deutschland GmbH, \\ Munich, Germany; ${ }^{6}$ Division of \\ Gastroenterology, University of \\ Alberta, Edmonton, AB, Canada
}

Correspondence: Jürgen Stein Interdisciplinary Crohn Colitis Center Rhein-Main, Schifferstr. 59, 60594

Frankfurt/Main, Germany

Tel +49699055978 10

Fax: +4969905597829

Email j.stein@em.uni-frankfurt.de
Background: Iron-deficiency anemia and iron deficiency are common comorbidities associated with inflammatory bowel disease (IBD) resulting in impaired quality of life and high health care costs. Intravenous iron has shown clinical benefit compared to oral iron therapy.

Aim: This study aimed to compare health care outcomes and costs after oral vs intravenous iron treatment for IBD patients with iron deficiency or iron deficiency anemia (ID/A) in Germany. Methods: IBD patients with ID/A were identified by ICD-10-GM codes and newly commenced iron treatment via ATC codes in 2013 within the InGef (formerly Health Risk Institute) research claims database. Propensity score matching was performed to balance both treatment groups. Non-observable covariates were adjusted by applying the difference-in-differences (DID) approach.

Results: In 2013, 589 IBD patients with ID/A began oral and 442 intravenous iron treatment. After matching, 380 patients in each treatment group were analyzed. The intravenous group had fewer all-cause hospitalizations (37\% vs $48 \%$ ) and ID/A-related hospitalizations (5\% vs $14 \%$ ) than the oral iron group. The 1-year preobservation period comparison revealed significant health care cost differences between both groups. After adjusting for cost differences by DID method, total health care cost savings in the intravenous iron group were calculated to be $€ 367$. While higher expenditure for medication $(€ 1,876)$ was observed in the intravenous iron group, the inpatient setting achieved most cost savings (€1,887).

Conclusion: IBD patients receiving intravenous iron were less frequently hospitalized and incurred lower total health care costs compared to patients receiving oral iron. Higher expenditures for pharmaceuticals were compensated by cost savings in other domains.

Keywords: inflammatory bowel disease, iron-deficiency anemia, claims data, iron treatment, health care cost, Germany

\section{Introduction}

Iron deficiency anemia (IDA) is the most common form of anemia worldwide ${ }^{1}$ and the most frequent extraintestinal complication of inflammatory bowel disease (IBD), a group of systemic inflammatory conditions including Crohn's disease and ulcerative colitis. $^{2-8}$ Although the cause of anemia in IBD is multifactorial, iron deficiency (ID) is one of the most prevalent underlying factors, caused by poor iron intake, chronic blood loss, or impaired absorption. ${ }^{9}$ ID without anemia may cause an array of clinical symptoms including fatigue, sleeping disorders, restless legs syndrome, attention deficit, discontentment, agitation, and female infertility. ${ }^{10,11}$ 
Diagnostic criteria for ID depend on the level of inflammation. In patients without clinical, endoscopic, or biochemical evidence of active disease, serum ferritin $<30 \mu \mathrm{g} / \mathrm{L}$ is an appropriate criterion. In the presence of inflammation, serum ferritin up to $100 \mu \mathrm{g} / \mathrm{L}$ may still be consistent with ID. ${ }^{12}$ The World Health Organization (WHO) currently defines anemia as a hemoglobin value $<12.0 \mathrm{~g} / \mathrm{dL}(7.45 \mathrm{mmol} / \mathrm{L})$ in non-pregnant women and $<13.0 \mathrm{~g} / \mathrm{dL}(8.07 \mathrm{mmol} / \mathrm{L})$ in men, or hematocrit levels of $36 \%$ and $39 \%$, respectively. ${ }^{13}$ This definition applies equally to patients with IBD. ${ }^{12,14}$

Chronic fatigue, diarrhea, abdominal pain, headache, dizziness, shortness of breath, and tachycardia are common symptoms of anemia in IBD patients and can have a strong influence on the quality of life as the ability to perform normal daily activities is reduced. ${ }^{11,15-17}$ Furthermore, symptoms of anemia are associated with psychological and social consequences and can influence health careassociated costs. ${ }^{18,19}$

IDA necessitates prompt management and treatment. ${ }^{5,12,20}$ However, IBD patients with IDA still receive iron therapy relatively infrequently. ${ }^{2,21-23}$ Effective treatment includes identifying and treating the underlying cause and initiating iron therapy with either oral or intravenous (IV) iron. The goal of iron therapy is to normalize hemoglobin levels and replete iron stores. ${ }^{12}$

Treatment options for IDA include oral and parenteral iron, erythropoiesis-stimulating agents, and blood transfusions. ${ }^{5}$ The administration of red blood cell transfusion should be considered carefully as there is evidence of adverse clinical outcomes regarding morbidity and mortality after blood transfusions. ${ }^{24}$ Only in cases where hemoglobin concentrations are below $7 \mathrm{~g} / \mathrm{dL}$ and in the presence of severe comorbidities and risk factors, or in life-threatening situations, should blood cell transfusions be considered. ${ }^{12}$

Compared to oral iron, IV iron treatment has shown medical benefits for IBD patients who have severe or refractory anemia, or during active disease..$^{5,9,12,25}$ However, as stated by Lee et al, only three studies have, to date, compared IV with oral iron replacement. ${ }^{26}$ Although all three studies showed favorable clinical outcomes for IV iron, there has been no discussion on the resulting complex economic impact. ${ }^{27}$

As mentioned earlier, ID affects a large number of patients with IBD, with an estimated prevalence from as low as $36 \%$ to as high as $90 \%$. A similarly wide range is reported for anemia in IBD patients, with reported rates spanning from $6 \%$ to $74 \%{ }^{9,28,29}$ Few prevalence estimates exist for Germany. ${ }^{8,21}$
However, Ott et al reported that, of the 279 patients with newly diagnosed IBD, 90 (32.3\%) had anemia at any point during the study period, of whom $70 \%$ met the diagnostic criteria for IDA. ${ }^{21}$

A number of studies have assessed the prevalence of iron deficiency and iron deficiency anemia (ID/A) in IBD, yet clinical evidence, and particularly economic evidence, concerning the comparison between oral and IV iron, is very limited in this field. Therefore, this study was conducted to fill the research gap of clinical and economic outcomes of patients with IBD and ID/A in Germany.

\section{Study objectives}

The primary objectives of this claims database analysis were to identify patients with IBD and ID/A newly treated with IV or oral iron therapy, to match oral iron-treated patients with IBD to IV iron-treated patients with IBD, and to compare health care resource use and health care costs of both treatment cohorts. Secondary objectives were to calculate the prevalence of ID/A in IBD, to identify potential undertreatment of IBD patients with ID/A, and to compare demographic characteristics of both treatment cohorts.

\section{Methods}

This retrospective claims data analysis was conducted using data from InGef, the German "Institut für angewandte Gesundheitsforschung, Berlin" (formerly known as the Health Risk Institute) research database. All data generated or analyzed during this study are included in this published article. The InGef research database comprises claims data from 75 of the 120 health insurance companies in Germany. The analysis sample includes the utilization and costs of services for $\sim 4$ million insured persons through 2014 on an anonymized individual level. This sample represents $4.8 \%$ of the German population and is considered representative of the total German population. ${ }^{30}$ Data from January 1, 2012, through September 30, 2014, were used for this analysis. The enrollment period for IBD patients with ID/A spanned from January 1, 2013, through December 31, 2013, to allow an individual pre-index period of four quarters (Q1/2012 to $\mathrm{Q} 3 / 2013)$ as well as an individual post-index period of the index quarter and three consecutive quarters (Q1/2013 to Q3/2014) (Figure 1).

The analysis included all patients in the database with a diagnosis of IBD identified by the International Statistical Classification of Diseases and Related Health Problems, 10th revision, German Modification (ICD-10-GM) codes K50* or K51* between January 2013 and December 2013. As no 


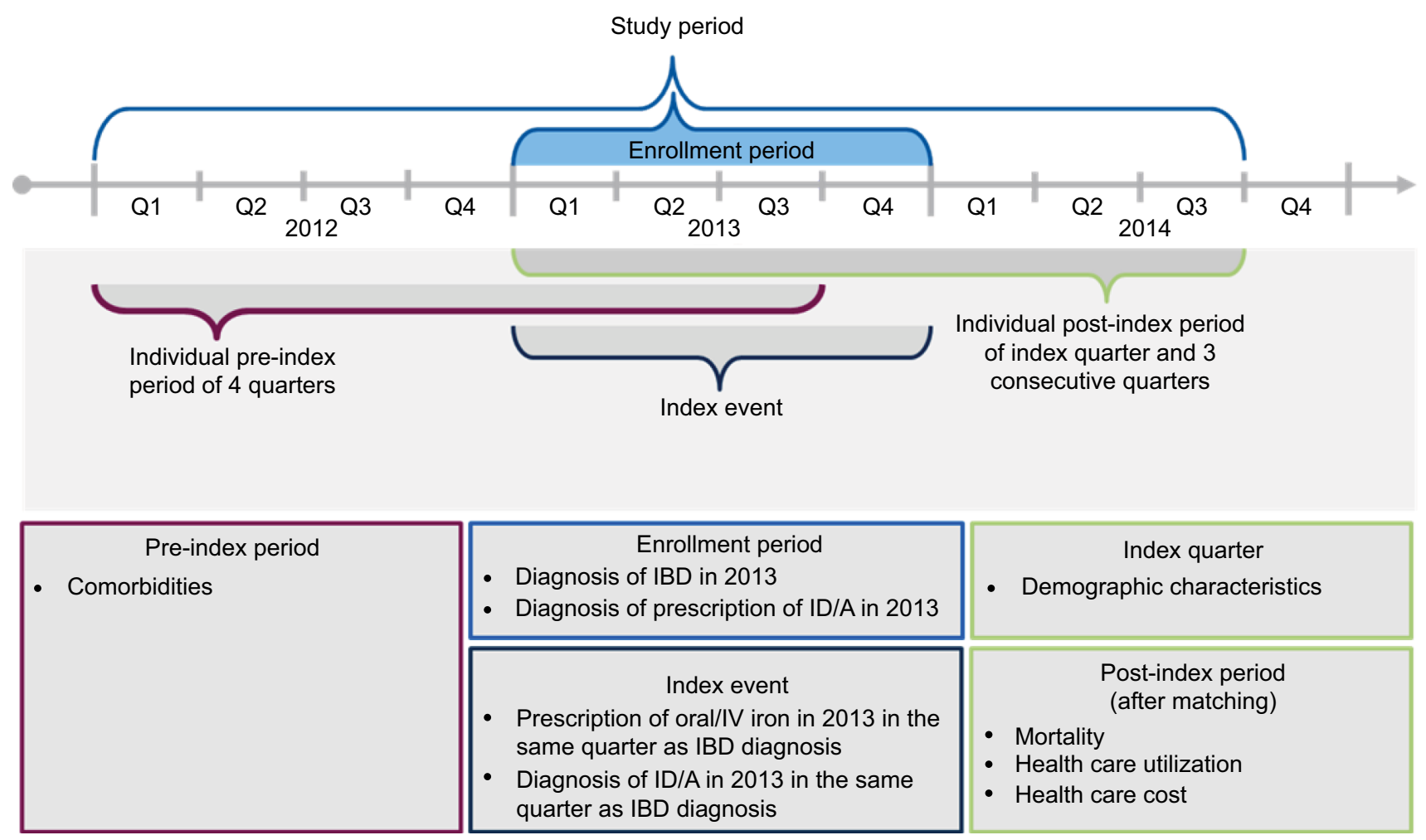

Figure I Study design.

Abbreviations: IBD, inflammatory bowel disease; ID/A, iron deficiency/iron deficiency anemia; IV, intravenous.

clinical data are available in claims data, the presence of ID and/or IDA was identified by ICD-10-GM codes D50* and E61.1 or iron treatment (via ATC codes) in the same timeframe. The individual pre-observation period was required to identify newly iron-treated patients.

\section{Ethics}

No ethical approval and consent were necessary as this is a retrospective database study based on anonymized claims data. The database is fully compliant with all data protection regulations in Germany and has been certified. Since the InGef (formerly HRI) research database includes verified accounting data of the participating insurance companies, these claims data are regularly audited by the insurance companies for reimbursement purposes and are prepared in accordance with German Social Law (paragraphs 287 SGB $\mathrm{V}$ and 75 SGB X). This study utilized an existing dataset in line with all data protection regulations and patients were not identified for the purpose of this study.

\section{Outcomes}

Patient characteristics were assessed by including demographics at the index quarter, and comorbidities were measured with the Deyo-Charlson comorbidity index in the pre-observation period of four quarters.

The outcomes consisted of health care resource use in terms of inpatient stays and sick leave - both all-cause and ID/Arelated - and mortality, during/after iron treatment. For the inpatient stays, ID/A-related visits were based on ICD-10-GM codes D50* or E61.1 as primary or secondary diagnosis.

Health care costs (expressed in $€$ ) were calculated within the observation period of the index quarter and three consecutive quarters, which included separate assessment of the sectors inpatient, outpatient, pharmaceuticals, devices and aids, and sick leave payments. Health care utilization and costs during treatment were analyzed using descriptive statistics.

\section{Matching}

Comparative treatment groups constituted IBD patients treated with oral iron vs IBD patients treated with IV iron, to analyze differences in all-cause mortality, health care utilization, and health care cost. A 1:1 propensity score matching was performed to directly balance both treatment groups. Propensity scores were calculated including the variables age, gender, and comorbidities, according to the updated Charlson Comorbidity Index (CCI) score, in a multivariate 
logistic regression. Matching couples were identified by applying the nearest neighbor-matching technique, and a caliper of width allowing 0.05 of the standard deviation of the propensity score was determined.

Absolute standardized differences of the two cohorts were calculated for the matching variables (age, gender, comorbidities according to CCI, CCI scores, length of stay, and other resource utilization variables) before and after matching to evaluate the matching performance of this technique.

The matching outcomes "all-cause mortality", "health care utilization", and "health care cost" between the two cohorts were compared with statistical tests ( $p$-values). The McNemar's test was applied for categorical variables and the Wilcoxon rank test was used for metric variables of twosided samples.

\section{DID in cost analysis}

Unobservable covariates were adjusted using the differencein-differences (DID) approach. When estimating the treatment effect, the treated units before and after treatment can be compared. However, this comparison might pick up effects of other factors that changed around the time of treatment. Therefore, a control group to "difference out" these con- founding factors and isolate the treatment effect is used. With the DID approach, the mean value of each group's outcome before and after treatment is taken into account and then the DIDs of the means are calculated. ${ }^{31}$ The DID method was applied in combination with propensity-score matching in many other studies, such as Zhou et al, Stokes et al, Stuart et al, and Gebel et al, showing that by adding the DID, a robust comparison of groups can be performed. ${ }^{32-35}$

\section{Results}

\section{Study population}

In total, 29,331 patients with IBD were identified in 2013. This overall study group was further stratified by the number of patients with ID/A, identified by the respective ICD10-GM codes, in the same quarter in 2013. A total of 3,561 IBD patients with ID/A were identified (12.1\%). Of these, 589 IBD patients with ID/A began oral and 442 IV iron treatment. Of the 2,379 patients with IBD and ID/A, over $50 \%$ $(n=1,348)$ revealed no prescription iron therapy at all in 2013. Bearing in mind the significant impact that even ID without anemia can have on quality of life, ${ }^{10}$ this implicit undertreatment may be considered cause for concern. Figure 2 depicts the sample selection process.
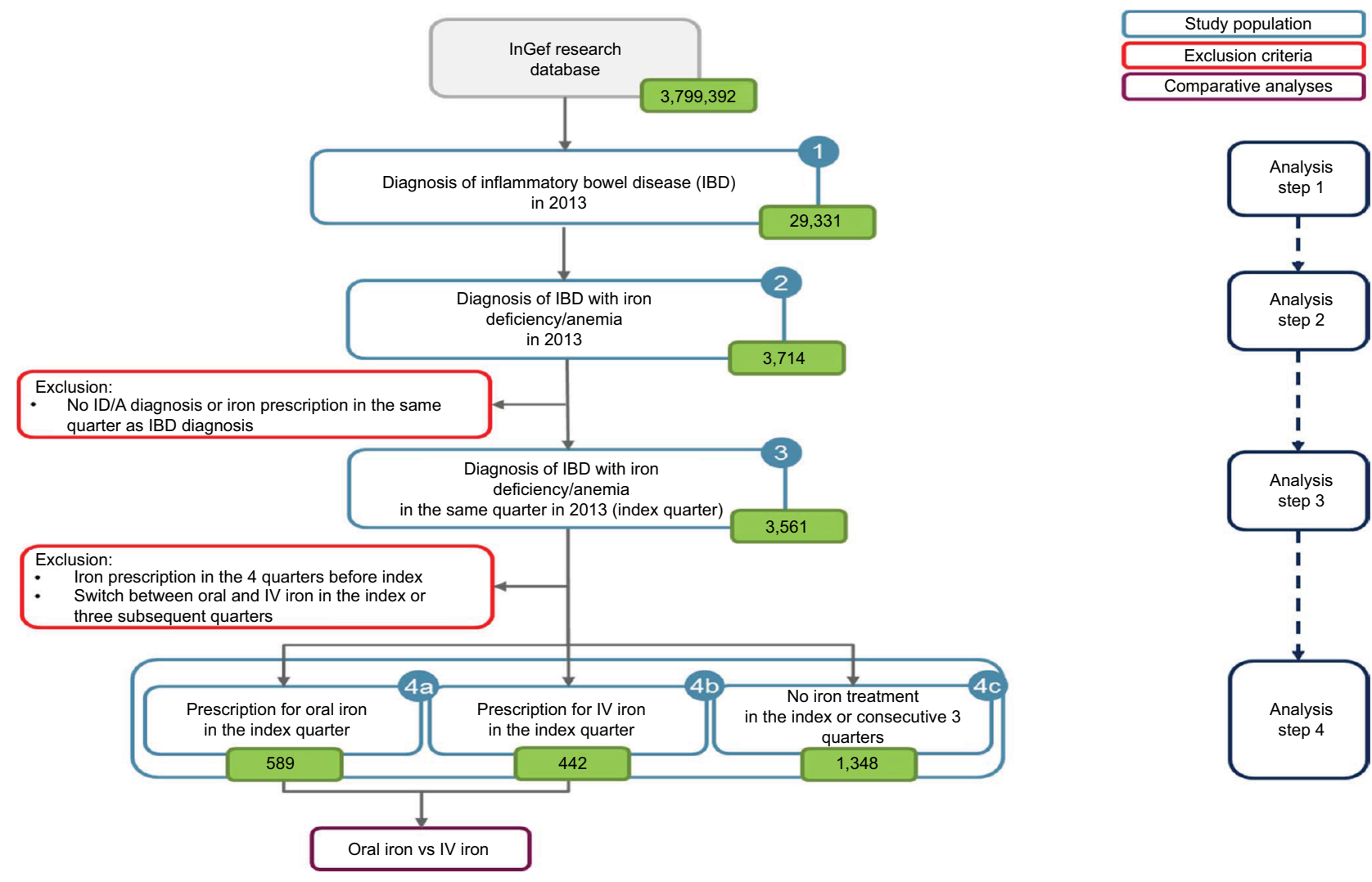

Figure 2 Sample selection.

Abbreviations: InGef, Institut für angewandte Gesundheitsforschung Berlin; IV, intravenous; ID/A, iron deficiency/iron deficiency anemia. 


\section{Patient characteristics}

Oral iron patients were almost 10 years older on average compared to patients newly treated with IV iron (50.13 vs 40.61 years). In addition, $37 \%$ vs $40 \%$ were male, respectively. Most IV iron-treated patients $(62.9 \%)$ received iron(III) hydroxide-polymaltose complex, followed by $24.9 \%$ who received iron(III)sodium-gluconate complex.

\section{After matching}

After matching for age, gender, and CCI scores, 380 patients were included in each treatment group. Mean age was adjusted for oral and IV iron patients (42.3 vs 42.2 years) and $67 \%$ vs $61 \%$ were female, respectively. Comorbidities were equally prevalent in both cohorts (Table 1).

\section{Health care resource use during/after iron treatment}

All-cause hospitalizations were significantly lower in the IV iron group compared to the oral iron cohort (37\% vs $48 \%$, $p=0.0019$ ). Also, fewer ID/A-related hospitalizations were observed in the IV group ( $5 \%$ vs $14 \%, p<0.001)$. Mean duration of all-cause hospitalizations was 8.5 days for the IV iron cohort and 7.9 days for the oral iron cohort ( $p=0.0016)$, whereas the duration of ID/A-related hospitalizations was considerably shorter for the IV cohort (7.0 vs 9.6 days, $p<0.001)$.

\section{Health care costs during/after iron treatment}

Mean total health care costs were significantly higher in the IV iron cohort ( $€ 13,365$ vs $€ 9,099, p<0.0001$ ), whereas inpatient costs were significantly lower for patients treated with IV iron ( $€ 2,796$ vs $€ 4,397, p=0.006)$ (Figure 3 ).

Total pharmaceutical costs were over twofold higher in the IV cohort compared to the oral iron cohort (€8,629 vs $€ 3,181)$. The higher medication costs in the IV iron-treated group were not caused by the IV iron preparations, which amounted to only $3.8 \%$ of the pharmaceutical cost. The highest proportion of these costs comprised prescriptions for biologic treatments $(58.8 \%)$. Table 2 depicts the mean pharmaceutical cost distribution of the respective treatment cohorts.

The significant health care cost differences could not be explained by the different iron treatments. Therefore, it was investigated whether baseline differences in the different cohorts may have been unobserved or unaccounted for, i.e., patient conditions that may have caused an outcome bias. Using the DID approach, the mean value of each group's

Table I Cohort characteristics after matching

\begin{tabular}{|c|c|c|}
\hline Patient characteristic & $\begin{array}{l}\text { Cohort I } \\
\text { Group 4a: oral iron treatment } \\
\mathbf{N}=380\end{array}$ & $\begin{array}{l}\text { Cohort } 2 \\
\text { Group } 4 \text { b: intravenous iron treatment } \\
N=380\end{array}$ \\
\hline \multicolumn{3}{|l|}{ Age } \\
\hline Mean & 42.33 & 42.17 \\
\hline SD & 17.39 & 17.16 \\
\hline Median & 40 & 40 \\
\hline Min & 12 & 12 \\
\hline Max & 92 & 89 \\
\hline \multicolumn{3}{|l|}{ Gender n (\%) } \\
\hline Male & $126(33.16)$ & $148(38.95)$ \\
\hline Female & $254(66.84)$ & $232(61.05)$ \\
\hline \multicolumn{3}{|l|}{ Comorbidities n (\%) } \\
\hline Cancer & $18(4.74)$ & $22(5.79)$ \\
\hline Chronic pulmonary disease & $74(19.47)$ & $67(17.63)$ \\
\hline Congestive heart failure & $14(3.68)$ & $12(3.16)$ \\
\hline Dementia & $<5$ (NA) & $<5$ (NA) \\
\hline Diabetes with complications & II (2.89) & II (2.89) \\
\hline Metastatic carcinoma & $<5$ (NA) & $<5$ (NA) \\
\hline Mild liver disease & $22(5.79)$ & $29(7.63)$ \\
\hline Paraplegia and hemiplegia & $7(1.84)$ & $<5$ (NA) \\
\hline Renal disease & $21(5.53)$ & $21(5.53)$ \\
\hline Rheumatic disease & $15(3.95)$ & $15(3.95)$ \\
\hline
\end{tabular}

Note: Patient counts below 5 are reported as $<5$ due to data protection regulations.

Abbreviations: SD, standard deviation; NA, not available. 


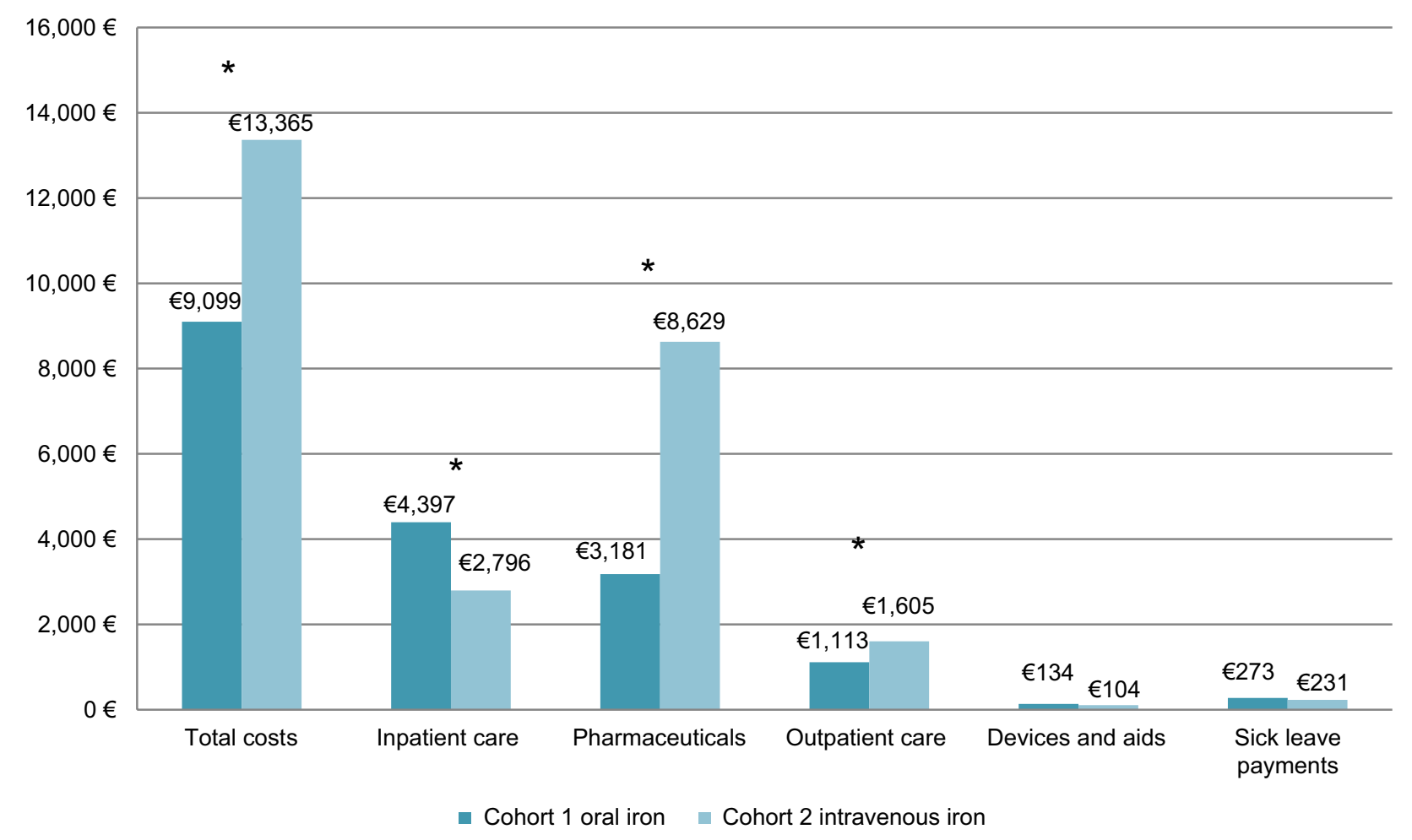

Figure 3 Health care cost during/after iron treatment.

Note: *Statistically significant differences $(p<0.05)$.

Table 2 Mean pharmaceutical cost distribution

\begin{tabular}{lll}
\hline Pharmaceutical type & $\begin{array}{l}\text { Cohort I } \\
\text { Group 4a: oral iron } \\
\text { treatment } \\
\mathbf{N = 3 8 0}\end{array}$ & $\begin{array}{l}\text { Cohort 2 } \\
\text { Group 4b: intravenous iron } \\
\text { treatment } \\
\mathbf{N = 3 8 0}\end{array}$ \\
\hline Oral iron & $32.5(1.0 \%)$ & $0(0.0 \%)$ \\
IV iron & $0(0.0 \%)$ & $327.0(3.8 \%)$ \\
Biologic treatment TNF alpha (ATC code L04AB*) & $1,518.4(47.7 \%)$ & $5,074.6(58.8 \%)$ \\
Other & $1,630.4(51.3 \%)$ & $3,226.3(37.4 \%)$ \\
Mean pharmaceutical cost (in $€)$ & $3,181.3(100 \%)$ & $8,628.8(100 \%)$ \\
\hline
\end{tabular}

Abbreviation: IV, intravenous.

outcome before and after treatment was applied to calculate the DID of the means.

\section{DID}

The assessment of incremental baseline differences after matching revealed significant health care cost differences in terms of outpatient care, pharmaceuticals, and total costs (Figure 4). Furthermore, no significant incremental differences were found in terms of all-cause or ID/A-related hospitalization in the pre-index period. The comparison of the means in the baseline period (Table 3) showed that the performed matching could not balance baseline differences in health care cost, as the incremental baseline total cost difference after matching was still $€ 4634.67$, suggesting bias due to unobserved variables/ heterogeneity, possibly related to unobservable differences in IBD severity.

To calculate the DID, pre-index and post-index outcomes were compared for the matched cohorts. Before treatment, patients in the IV iron cohort had higher costs in all assessed health care cost sectors. After/during treatment (post-index period), however, lower costs were observed for inpatient care, devices, and aids as well as sick leave payments compared to patients on oral treatment. 


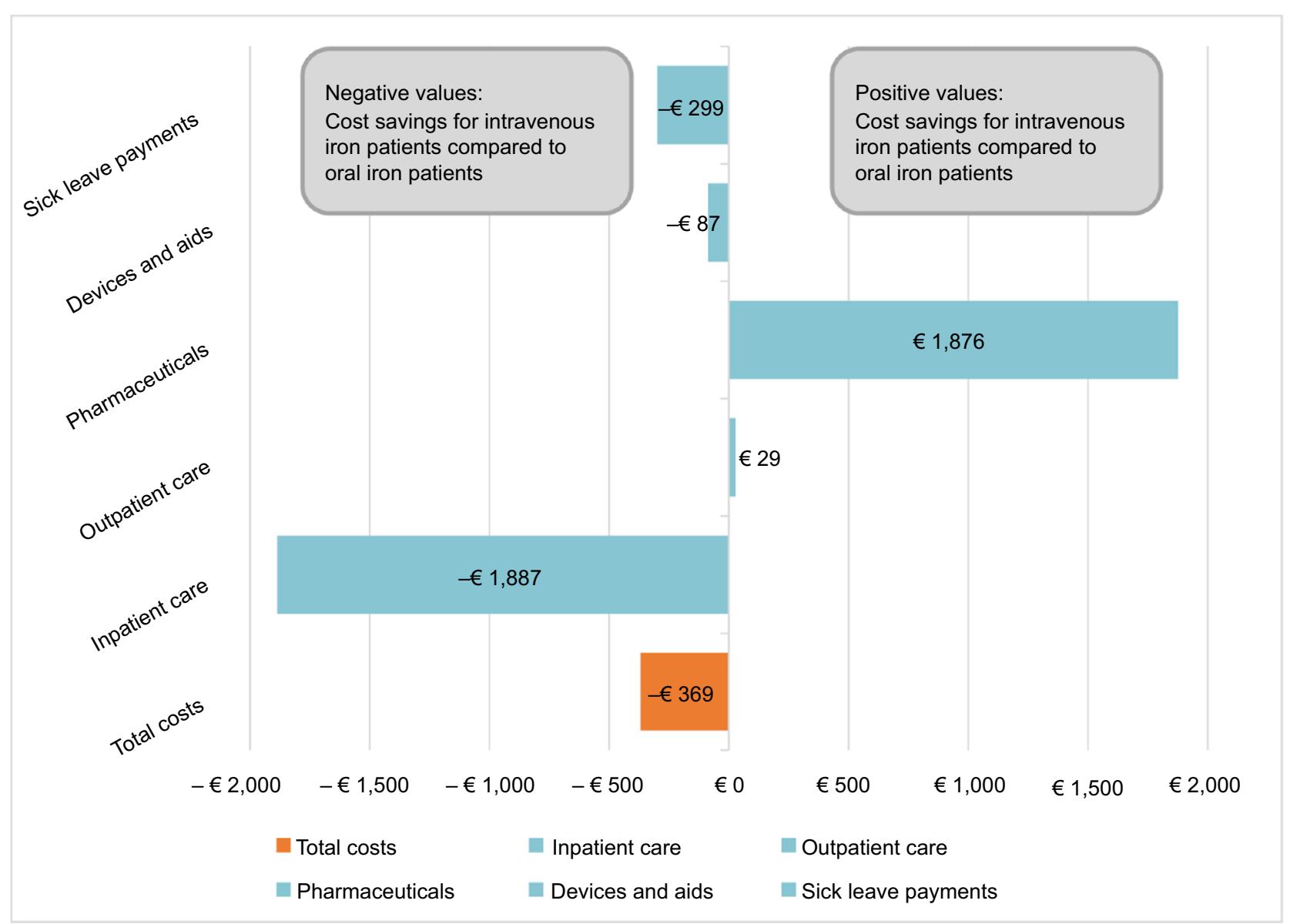

Figure 4 Difference-in-differences approach.

Table 3 Comparison of mean baseline outcomes after matching

\begin{tabular}{|c|c|c|c|c|}
\hline Healthcare cost sector & $\begin{array}{l}\text { Cohort I } \\
\text { Group 4a: oral iron } \\
\text { treatment } \\
\mathbf{N}=\mathbf{3 8 0}\end{array}$ & $\begin{array}{l}\text { Cohort } 2 \\
\text { Group } 4 \mathrm{~b} \text { : intravenous } \\
\text { iron treatment } \\
\mathrm{N}=380\end{array}$ & $\begin{array}{l}\text { Incremental } \\
\text { difference }\end{array}$ & $p$-value \\
\hline Inpatient care (in €) & $2,701.29$ & $2,986.90$ & 285.61 & 0.4226 \\
\hline Outpatient care (in $€$ ) & 880.36 & $1,343.13$ & 462.77 & $<0.001$ \\
\hline Pharmaceuticals (in €) & $2,3 \mid 4.63$ & $5,886.21$ & $3,571.58$ & $<0.001$ \\
\hline Devices and aids (in $€$ ) & 61.92 & 119.01 & 57.09 & 0.1892 \\
\hline Sick leave payments (in €) & 109.42 & 367.06 & 257.64 & 0.0607 \\
\hline Total costs (in $€$ ) & $6,067.63$ & $10,702.30$ & 4634.67 & $<0.001$ \\
\hline
\end{tabular}

After adjusting for expenditure differences by DID, total health care cost savings per patient in the IV iron group were calculated to be $€ 369$. While expenditure on pharmaceuticals was higher in the IV iron group $(€ 1,876)$, the inpatient setting achieved most cost savings $(€ 1,887)$. The calculated differences for the pre-index and post-index period as well as the DID are shown in Table 4.

\section{Discussion}

This retrospective German claims data analysis is the first comprehensive analysis of this type to compare different iron treatments in IBD patients in the German health care system. The underlying data sample of this study comprises health insurances operating nationwide and is representative in terms of age and gender of the German population. The 
Table 4 Difference-in-differences approach

\begin{tabular}{|c|c|c|c|}
\hline Expenditure sector & $\begin{array}{l}\text { Pre-index period } \\
\text { Intravenous-oral } \\
\text { (after matching) } \\
\mathbf{N}=380\end{array}$ & $\begin{array}{l}\text { Post-index period } \\
\text { Intravenous-oral } \\
\text { (after matching) } \\
\mathbf{N}=380\end{array}$ & $\begin{array}{l}\text { Difference-in- } \\
\text { differences } \\
\text { (post-index period- } \\
\text { pre-index period) }\end{array}$ \\
\hline Inpatient care (in $€$ ) & 285.61 & $-1,601.02$ & $-1,886.63$ \\
\hline Outpatient care (in $€$ ) & 462.77 & 491.49 & 28.72 \\
\hline Pharmaceuticals (in $€$ ) & $3,571.58$ & $5,447.5 I$ & $1,875.93$ \\
\hline Devices and aids (in €) & 57.09 & -30.06 & -87.15 \\
\hline Sick leave payments (in $€$ ) & 257.64 & -41.82 & -299.46 \\
\hline Total costs (in €) & $4,634.67$ & $4,266.09$ & -368.58 \\
\hline
\end{tabular}

InGef database correlates well with German reference data with respect to hospitalization rates, overall mortality rate, and prescription rates for the 20 most frequently reimbursed drug classes. ${ }^{30}$ Hence, the database can be considered to have external validity and the results represent real-world evidence for the German state health insurance (SHI) population.

In our study, the prevalence of IBD patients with ID/A was $\sim 12 \%$, taking an ID/A and IBD diagnosis in the same quarter to be a proxy for related disease. For a diagnosis coded within the same calendar year, the prevalence was $12.6 \%$. Our prevalence was within the lower range of prevalence rates reported in other studies. In a review by Gisbert and Gomollon, the frequency of anemia ranged from 16\% in outpatients to $68 \%$ in hospitalized patients. ${ }^{36}$ Based on the available studies, mean prevalences of $45 \%$ for ID and $17 \%$ for IDA in IBD were calculated, ${ }^{36}$ whereas Kulnigg and Gasche estimated a prevalence interval of anemia between $6 \%$ and $74 \%$ in their systematic review. ${ }^{29}$ Ershler et al analyzed the largest sample size, including 7,200 IBD patients, and reported findings comparable to our study, with anemia occurring in about $13 \%$ of IBD patients. ${ }^{37}$ Reasons for the observed variances in the literature concerning the prevalence of ID and/or anemia are differences in inclusion and exclusion criteria, patient populations, considered settings, and study sizes. In general, it should be noted that our study considered both ID/A, which in turn might limit the comparability of the prevalence estimates.

In our study, patients treated with IV iron were on average 10 years younger than patients receiving oral iron treatment, and $40 \%$ were male. These findings are in line with Bager and Dahlerup, who reported a mean age of 41 years at the time of the first iron dose and a percentage of $45 \%$ males in a sample of IBD patients treated with IV iron in Denmark. ${ }^{19}$

Furthermore, the health care utilization analysis showed that patients treated with IV iron incurred significantly fewer hospitalizations in terms of both all-cause (37\% vs $48 \%$, $p=0.0019)$ and ID/A-related hospitalizations (5\% vs $14 \%$, $p<0.001$ ), suggesting that IV iron therapy is associated with improved health care outcomes. As found by Baumgart and le Claire in their analysis of IBD patients in a German hospital, many patients experienced disease- and treatmentrelated complications such as anemia due to bleeding and/or ID, which were coded as secondary diagnoses. ${ }^{27}$ Our study has shown that if patients are treated with IV iron therapy, then both all-cause and ID/A-related hospitalizations of IBD patients will be reduced.

The performed propensity-score matching was evaluated by calculating incremental differences for health care utilization and health care costs in the pre-index period. Baseline health care costs after matching showed significant incremental cost differences in terms of outpatient care $(p<0.001)$, pharmaceuticals $(p<0.001)$, and total costs $(p<0.001)$. Consequently, the performed matching could not balance baseline differences in health care costs, suggesting bias due to unobserved variables or heterogeneity, such as IBD severity which was not observable on the basis of the available data. DID is a validated method used to take account of unobservable differences in baseline variables. When applying DID, it is assumed that parallel trends occur, implying that trends in the outcomes between the treatment groups are equal before the intervention was implemented. In addition, unexpected or unpredictable events and spillover effects which depend, for example, on policy, are assumed to affect both observed treatment groups equally. ${ }^{31}$ This technique was adopted by Zhou et al, who applied the DID approach in a comparative effectiveness analysis in order to compare two unbalanced cohorts after propensity-score matching. Their study showed that DID offered a more adequate comparison than propensity-score matching. ${ }^{32}$ Stokes et al applied DID when analyzing hospital admission data stratified by risk level. ${ }^{33}$ The study groups of both Patel et al and Rajaram et al applied the DID approach by using the Medicare US claims 
database and the ACS NSQIP (American College of Surgeons National Surgical Quality Improvement Program) registry of the ACGME (Accreditation Council for Graduate Medical Education) to control for background trends in measuring health-related outcomes of patients. ${ }^{38,39}$ In the present study, to calculate the DID, pre-index and post-index outcomes were compared for the matched cohorts. ${ }^{31}$ After adjusting for expenditure differences by DID, total per patient health care cost savings of $€ 369$ were calculated in the IV iron group. While higher expenditures occurred in the IV iron group for pharmaceuticals (€1,876) and outpatient care (€29), the inpatient setting achieved most cost savings (€1,887), followed by savings in sick leave payments (€300), and devices and aids (€87).

\section{Limitations}

Claims databases constitute a suitable tool for studies analyzing epidemiological measures, health care utilization and health care costs, as data are recorded independently of any study purposes or clinical research recruiting participants. Nevertheless, claims data analyses present limitations that need to be addressed. One limitation is that administrative data are primarily collected for accounting purposes and not for clinical or research purposes. Therefore, clinical parameters including lab tests are not recorded in the database, thus precluding the additional verification of ID/A diagnosis via laboratory parameters such as hemoglobin or ferritin values. Furthermore, diagnoses in the outpatient setting are only available on a quarterly basis so that the exact date of diagnosis cannot be elucidated. In addition, only the first year following the start of iron therapy was analyzed for outcome measures. Long-term effects on clinical outcome and health care costs several years after iron treatment initiation could, therefore, not be considered.

A general limitation of claims data analyses in assessing medication intake is the inability to observe whether actual intake and dosage of medications were in accordance with the package insert. In addition, off-label and nonreimbursed prescription drugs are not recorded in claims data. Furthermore, the indication for the prescription is not captured. The analyses are, therefore, based on assumptions about the behavior of the identified patients, which in turn might lead to under-/overestimation of relevant endpoints. Additionally, other important cost factors, e.g., costs of time lost from work, are difficult to assess from claims data. As the data analyzed in this study derive from statutory health insurance companies only, the private sector was not included.
Concerning the performed matching, it cannot be ruled out that unobserved variables were unequally distributed in both groups, leading to biased results. Furthermore, the study cohorts constituted relatively small sample sizes that must be considered when interpreting the study findings. In addition, with the DID approach, it is assumed that parallel trends occur, i.e., trends in the outcomes between the observed treatment groups are the same before the intervention was implemented. Furthermore, it is assumed that unexpected or unpredictable events affect both treatment groups equally, which in turn, may limit a calculated cost benefit for the IV iron group. ${ }^{31}$

\section{Conclusion}

A total of 29,331 IBD patients were identified in the database analysis, of whom $\sim 15 \%$ had confirmed ID/A. Over $50 \%$ of IBD patients with ID/A received no prescription iron replacement therapy. Patients who received IV iron treatment were on average 10 years younger than patients on oral iron, and $40 \%$ were male.

Analysis of health care utilization costs during the year following therapy initiation showed that IBD patients receiving IV iron treatment incurred fewer all-cause and ID/A-related hospitalizations and had lower total health care expenditure compared to patients treated with oral iron. Higher pharmaceutical costs in the group of patients treated with IV iron, comprising a minor share for the iron treatment itself, were compensated by cost savings in other health care sectors when compared to patients treated with oral iron. Further studies are needed to examine the long-term effect of IV vs oral iron therapy on health care utilization costs in IBD patients with ID/A.

\section{Acknowledgment}

The data analysis was performed in cooperation between Xcenda and Elsevier Health Analytics. Vifor Pharma has a research consultancy agreement with Xcenda and funded the conduct of the data analysis. The authors thank Janet Collins (iCCC Rhein-Main, Frankfurt/Main, Germany) for proofreading the manuscript.

\section{Disclosure}

SHO, TH, EL, KN, and DF are employees of and hold stock/ stock options in Vifor Pharma. JS and DCB have received reimbursements for participation in the scientific advisory council for this study from Vifor Pharma. JS has received lecturing fees from Vifor Pharma. JSH, KB, and SB are employees of Xcenda, which has a research consultancy 
agreement with Vifor Pharma. The authors report no other conflicts of interest in this work.

\section{References}

1. Camaschella C. Iron-deficiency anemia. N Engl J Med. 2015;372(19): 1832-1843.

2. Blumenstein I, Dignass A, Vollmer S, Klemm W, Weber-Mangal S, Stein J. Current practice in the diagnosis and management of IBD-associated anaemia and iron deficiency in Germany: the German AnaemIBD Study. J Crohns Colitis. 2014;8(10):1308-1314.

3. Danese S, Hoffman C, Vel S, et al. Anaemia from a patient perspective in inflammatory bowel disease: results from the European Federation of Crohn's and Ulcerative Colitis Association's online survey. Eur J Gastroenterol Hepatol. 2014;26 (12):1385-1391.

4. Rogler G, Vavricka S. Anemia in inflammatory bowel disease: an underestimated problem? Front Med. 2014;1:58.

5. Martin J, Radeke HH, Dignass A, Stein J. Current evaluation and management of anaemia in patients with inflammatory bowel disease. Expert Rev Gastroenterol Hepatol. 2017;11(1):19-32.

6. Lupu A, Diculescu M, Diaconescu R, et al. Prevalence of anemia and iron deficiency in Romanian patients with inflammatory bowel disease: a prospective multicenter study. $J$ Gastrointestin Liver Dis. 2015;24(1):15-20.

7. Koutroubakis IE, Ramos-Rivers C, Regueiro M, et al. Persistent or recurrent anemia is associated with severe and disabling inflammatory bowel disease. Clin Gastroenterol Hepatol. 2015;13(10):1760-1766.

8. Filmann N, Rey J, Schneeweiss S, et al. Prevalence of anemia in inflammatory bowel diseases in European countries: a systematic review and individual patient data meta-analysis. Inflamm Bowel Dis. 2014;20(5):936-945.

9. Stein J, Hartmann F, Dignass AU. Diagnosis and management of iron deficiency anemia in patients with IBD. Nat Rev Gastroenterol Hepatol. 2010;7(11):599-610.

10. Pratt JJ, Khan KS. Non-anaemic iron deficiency - a disease looking for recognition of diagnosis: a systematic review. Eur J Haematol. 2016;96(6):618-628.

11. Agarwal R. Nonhematological benefits of iron. Am J Nephrol. 2007;27(6):565-571.

12. Dignass AU, Gasche C, Bettenworth D, et al. European consensus on the diagnosis and management of iron deficiency and anaemia in inflammatory bowel diseases. J Crohns Colitis. 2015;9(3):211-222.

13. World Health Organization (WHO). Iron Deficiency Anemia: Assessment, Prevention and Control - A Guide for Programme Managers. Geneva: WHO; 2001.

14. Van Assche G, Dignass A, Bokemeyer B, et al. Second European evidence-based consensus on the diagnosis and management of ulcerative colitis part 3: special situations. J Crohns Colitis. 2013;7(1):1-33.

15. Bager P, Befrits R, Wikman O, et al. Fatigue in out-patients with inflammatory bowel disease is common and multifactorial. Aliment Pharmacol Ther. 2012;35(1):133-141.

16. Bager P, Befrits R, Wikman O, et al. High burden of iron deficiency and different types of anemia in inflammatory bowel disease outpatients in Scandinavia: a longitudinal 2-year follow-up study. Scand J Gastroenterol. 2013;48(11):1286-1293.

17. Wells CW, Lewis S, Barton JR, Corbett S. Effects of changes in hemoglobin level on quality of life and cognitive function in inflammatory bowel disease patients. Inflamm Bowel Dis. 2006;12(2):123-130.

18. Gasche C, Lomer MC, Cavill I, Weiss G. Iron, anaemia, and inflammatory bowel diseases. Gut. 2004;53(8):1190-1197.

19. Bager P, Dahlerup JF. The health care cost of intravenous iron treatment in IBD patients depends on the economic evaluation perspective. J Crohns Colitis. 2010;4(4):427-430.
20. Goldberg ND. Iron deficiency anemia in patients with inflammatory bowel disease. Clin Exp Gastroenterol. 2013;6:61-70.

21. Ott C, Liebold A, Takses A, Strauch UG, Obermeier F. High prevalence but insufficient treatment of iron-deficiency anemia in patients with inflammatory bowel disease: results of a population-based cohort. Gastroenterol Res Pract. 2012;2012:595970.

22. Voegtlin M, Vavricka SR, Schoepfer AM, et al. Prevalence of anaemia in inflammatory bowel disease in Switzerland: a cross-sectional study in patients from private practices and university hospitals. J Crohns Colitis. 2010;4(6):642-648.

23. Stein J, Bager P, Befrits R, et al. Anaemia management in patients with inflammatory bowel disease: routine practice across nine European countries. Eur J Gastroenterol Hepatol. 2013;25(12):1456-1463.

24. Murphy GJ, Reeves BC, Rogers CA, Rizvi SI, Culliford L, Angelini GD. Increased mortality, postoperative morbidity, and cost after red blood cell transfusion in patients having cardiac surgery. Circulation. 2007;116(22):2544-2552.

25. Kim KO. Management of anemia in patients with inflammatory bowel disease. Korean J Gastroenterol. 2015;65(3):145.

26. Lee TW, Kolber MR, Fedorak RN, van Zanten SV. Iron replacement therapy in inflammatory bowel disease patients with iron deficiency anemia: a systematic review and meta-analysis. J Crohns Colitis. 2012;6(3):267-275.

27. Baumgart DC, le Claire M. The expenditures for academic inpatient care of inflammatory bowel disease patients are almost double compared with average academic gastroenterology and hepatology cases and not fully recovered by diagnosis-related group (DRG) proceeds. PLoS One. 2016;11(1):e0147364.

28. Wilson A, Reyes E, Ofman J. Prevalence and outcomes of anemia in inflammatory bowel disease: a systematic review of the literature. $\mathrm{Am}$ J Med. 2004;116 (Suppl 7A):44S-49S.

29. Kulnigg S, Gasche C. Systematic review: managing anaemia in Crohn's disease. Aliment Pharmacol Ther. 2006;24(11-12):1507-1523.

30. Andersohn F, Walker J. Characteristics and external validity of the German Health Risk Institute (HRI) Database. Pharmacoepidemiol Drug Saf. 2016;25(1):106-109.

31. Dimick JB, Ryan AM. Methods for evaluating changes in health care policy: the difference-in-differences approach. JAMA. 2014;312(22): 2401-2402.

32. Zhou H, Taber C, Arcona S, Li Y. Difference-in-differences method in comparative effectiveness research: utility with unbalanced groups. Appl Health Econ Health Policy. 2016;14(4):419-429.

33. Stokes J, Kristensen SR, Checkland K, Bower P. Effectiveness of multidisciplinary team case management: difference-in-differences analysis. BMJ Open. 2016;6(4):e010468.

34. Stuart EA, Huskamp HA, Duckworth K, et al. Using propensity scores in difference-in-differences models to estimate the effects of a policy change. Health Serv Outcomes Res Methodol. 2014;14(4): 166-182.

35. Gebel M, Vossemer J. The impact of employment transitions on health in Germany. A difference-in-differences propensity score matching approach. Soc Sci Med. 2014;108:128-136.

36. Gisbert JP, Gomollon F. Common misconceptions in the diagnosis and management of anemia in inflammatory bowel disease. Am J Gastroenterol. 2008;103(5):1299-1307.

37. Ershler WB, Chen K, Reyes EB, Dubois R. Economic burden of patients with anemia in selected diseases. Value Health. 2005;8(6):629-638.

38. Patel MS, Volpp KG, Small DS, et al. Association of the 2011 ACGME resident duty hour reforms with mortality and readmissions among hospitalized medicare patients. JAMA. 2014;312(22):2364-2373.

39. Rajaram R, Chung JW, Jones AT, et al. Association of the 2011 ACGME resident duty hour reform with general surgery patient outcomes and with resident examination performance. JAMA. 2014;312(22):2374-2384. 
ClinicoEconomics and Outcomes Research is an international, peerreviewed open-access journal focusing on health technology assessment, pharmacoeconomics and outcomes research in the areas of diagnosis, medical devices, and clinical, surgical and pharmacological intervention. The economic impact of health policy and health systems organization also constitute important areas of coverage. The manuscript management system is completely online and includes a very quick and fair peer-review system, which is all easy to use. Visit http://www.dovepress.com/testimonials.php to read real quotes from published authors.

Submit your manuscript here: https://www.dovepress.com/clinicoeconomics-and-outcomes-research-journal 